\title{
SUPERFÍCIE FOLIAR DA VIDEIRA 'FOLHA DE FIGO' E 'NIAGARA ROSADA' CONDUZIDA EM DIFERENTES SISTEMAS DE CONDUÇÃO ${ }^{1}$
}

\author{
Surface leaf of the vine 'Leaf of Fig' and 'Rosy Niagara' grown in different culture systems \\ Paulo Márcio Norberto ${ }^{2}$, Murillo de Albuquerque Regina ${ }^{3}$, Nilton Nagib Jorge Chalfun ${ }^{4}$, \\ Ângela Maria Soares ${ }^{5}$, Vanessa Brito Fernandes ${ }^{6}$, Evandro Bordignon Gajego ${ }^{7}$
}

\begin{abstract}
RESUMO
Os efeitos da interação entre a forma do dossel vegetal e os fatores ambientais locais resultam no microclima que determinará alguns aspectos fisiológicos da videira, podendo afetar significativamente o crescimento vegetativo da videira, a produtividade do vinhedo, bem como a qualidade da uva e do vinho. O trabalho foi desenvolvido na Estação de Viticultura e Enologia de Caldas EPAMIG, Caldas, MG, com o objetivo de avaliar o efeito do sistema de condução sobre a superfície foliar da videira (Vitis labrusca L.), durante as safras 2003, 2004 e 2005. As variedades produtoras utilizadas no experimento foram 'Niágara Rosada' para mesa e 'Folha de Figo' para produção de vinhos; como porta - enxerto utilizou-se o '420-A'. O delineamento experimental foi DIC em fatorial 4x2 e os sistemas adotados foram cordão simples, latada, espaldeira e lira. Determinaram-se as seguintes variáveis: superfície foliar primária, secundária, total e superfície foliar exposta (SFE).Verificou-se que o sistema cordão simples conferiu a menor superfície foliar total em ambas as variedades, em todas safras avaliadas. Tanto o sistema espaldeira quanto o cordão simples conferiram à menor superfície foliar exposta em ambas as variedades e os sistemas latada e lira, os maiores valores de (SFE).
\end{abstract}

Termos para indexação: Videira, Superfície Foliar, Sistema de condução, Vitis labrusca.

\section{ABSTRACT}

The effects of the interaction between the form of the vegetable canopy and the environmental factors places result in the microclima that will determine some physiologic aspects of the vine, and may affect significantly the vegetative growth of the vine, the productivity of the vineyard, as well as the quality of the grape and wine. The work was developed in the Station of Viticulture and Enologia of Caldas EPAMIG, Caldas, MG, with the aim of evaluating the effect of the Training system on the foliar surface of the vine (Vitis labrusca L.) during the crops 2003, 2004 and 2005. The producing varieties used in the experiment were 'Niágara Rosy' for table and 'Leaf of Figs' for production of wines; as Door - I graft, the was used ' 420-A '. The experimental delineate was DIC in factorial $4 \times 2$ and the adopted systems were simple string, trellis, espaldeira and lira. One determined the following variables: primary foliar surface, secondary, total and exposed foliar surface (SFE). One verified that the simple string system checked the smallest total foliar surface in both varieties, in all appraised crops. As much the espaldeira system as the simple string checked the smallest foliar surface exposed in both varieties and the systems trellis and lira, the largest values of (SFE).

Index terms: Vine, Surface to Foliar, Training System, Vitis labrusca.

(Recebido em 15 de fevereiro de 2007 e aprovado em 22 de maio de 2007)

\section{INTRODUÇÃO}

O sistema de condução da videira é uma das técnicas aplicadas que contribuem para definir a forma da planta, modificando as condições microclimáticas como temperatura das folhas, umidade e intensidade de radiação, entre outras, no interior da copa da videira (CARBONNEAU, 1991; CASTRO, 1989).
Ele influencia na distribuição e orientação das folhas do dossel vegetativo e, conseqüentemente, na interceptação da radiação solar (CHAMPAGNOL, 1984). A largura do sistema de condução e o nível de desenvolvimento vegetativo da videira condicionam a distribuição dos cachos e das folhas no dossel; portanto, a captação da energia luminosa é feita de forma diferenciada em razão das características inerentes a cada sistema. A

\footnotetext{
${ }^{1}$ Parte da tese de Doutorado do primeiro autor apresentada à Universidade Federal de Lavras/UFLA, Cx. P. 3037 - $37200-000$ - Lavras, MG. 2Doutor - Empresa de Pesquisa Agropecuária de Minas Gerais/EPAMIG - Br 494, Km 02 - 36301-360 - São João Del Rei, MG - paulonor@ufla.br 3Ph. D., Pesquisador - Empresa de Pesquisa Agropecuária de Minas Gerais/EPAMIG-FECD - Cx. P. 33 - 37780-000 - Caldas, MG - murillo@epamigcaldas.br ${ }^{4}$ Doutor, Professor Titular - Departamento de Agricultura/DAG - Universidade Federal de Lavras/UFLA - Cx. P. 3037 - $37200-000$ - Lavras, MG nagib@ufla.br

${ }_{5}^{5}$ Doutora, Professora Adjunto - Departamento de Biologia/DBI - Universidade Federal de Lavras/UFLA - Cx. P. 3037 - $37200-000$ - Lavras, MG amsoares@ufla.br

${ }^{6}$ Mestre em Estatística - Departamento de Ciências Exatas/DEX - Universidade Federal de Lavras/UFLA - Cx. P. 3037 - $37200-000$ - Lavras, MG vanessabrito@ufla.br - Bolsista do CNPq/UFLA

${ }^{7}$ Graduando em Agronomia - Departamento de Agricultura/DAG - Universidade Federal de Lavras/UFLA - Cx. P. 3037 - $37200-000$ - Lavras, MG gajego@ufla.br
} 
penetração da radiação solar no dossel vegetativo favorece a iniciação floral, a fertilidade da gema, o pegamento do fruto e a maturação da uva (CARBONNEAU, 1982). Além disso, influencia o desenvolvimento da planta e a síntese de compostos orgânicos, melhorando a produção e a qualidade da uva (SMART, 1985).

Nogueira (1984) relata que na região sul de Minas Gerais as condições climáticas na época de maturação da uva não são adequadas, especialmente por causa da alta precipitação e elevada nebulosidade, reduzindo a qualidade da uva e, por conseqüência, a do vinho. Contudo, acredita-se que o uso correto do sistema de condução pode minimizar os efeitos negativos do excesso de chuvas e nebulosidade sobre a qualidade da uva, por propiciar condições microclimáticas mais favoráveis e assim, melhorar a qualidade do produto final. Objetivouse no presente trabalho avaliar o efeito de diferentes sistemas de condução, sobre a superfície foliar da videira (Vitis labrusca L.) 'Niágara Rosada'e 'Folha de Figo', em diferentes fases fenológicas.

\section{MATERIAL E MÉTODOS}

O trabalho foi desenvolvido no Núcleo Tecnológico Epamig Uva e Vinho da Empresa de Pesquisa Agropecuária de Minas Gerais (EPAMIG), no município de Caldas, MG, situado a $1150 \mathrm{~m}$ de altitude, com temperatura média anual de $19^{\circ} \mathrm{C}$, umidade relativa de $75 \%$ e precipitação pluviométrica de 1500mm anuais, durante as safras 2003, 2004 e 2005.

As variedades produtoras utilizadas no experimento foram 'Niágara Rosada' e 'Folha de Figo' (sinonímia regional da variedade 'Bordô' ou 'Ives'), enxertadas sobre o porta - enxerto '420-A', todas oriundas do banco de germoplasma da EPAMIG.

O delineamento experimental empregado foi inteiramente casualizado em fatorial $4 \times 2$, ou seja, quatro sistemas de condução e duas variedades, com quatro repetições por parcela, perfazendo um total de 32 plantas.

A análise de variância foi realizada através do teste $\mathrm{F}$ e o teste utilizado para comparação das médias foi o ScottKnott, a 5\% de probabilidade, para todas as características.

Características avaliadas nas safras 2003, 2004 e 2005:

\section{Superfície foliar primária, secundária, total e exposta.}

Ao longo do período experimental foram feitas avaliações da superfície foliar primária, secundária e total nas plantas, durante a fase fenológica de final de maturação 'pintor', ocorrida no mês de dezembro nas três safras avaliadas, em que:

A)- A superfície foliar primária foi mensurada escolhendo-se ao acaso dois ramos situados na porção mediana dos ramos produtivos, em quatro plantas, para cada sistema de condução. As medidas foram feitas em, aproximadamente, $30 \%$ do total de folhas, considerando áreas sombreadas e não sombreadas. Inicialmente foram tomadas as medidas das nervuras laterais (L2 esquerda e direita), conforme Figura 1, levando-se em consideração os dois lados do ramo devido ao fato de os ramos da videira apresentarem crescimento em forma de espiral. As primeiras nervuras L2 consideradas situavam-se na folha (n), internamente e oposta ao cacho, e as folhas seguintes a serem consideradas foram as de $n+4$ e $n+8$; mudando-se de lado, consideraram-se as de $\mathrm{n}+11, \mathrm{n}+$ $15, n+19$; e retornando-se ao lado inicial, as de $n+22, n$ + 30 (Figura 2), de acordo com a metodologia de Carbonneau (1976).

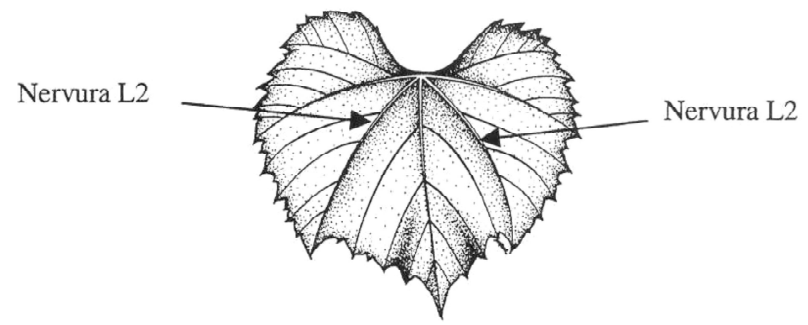

Figura 1 - Folha de videira com detalhe das nervuras L2 esquerda e direita. Fonte: Pommer et al. (1997).

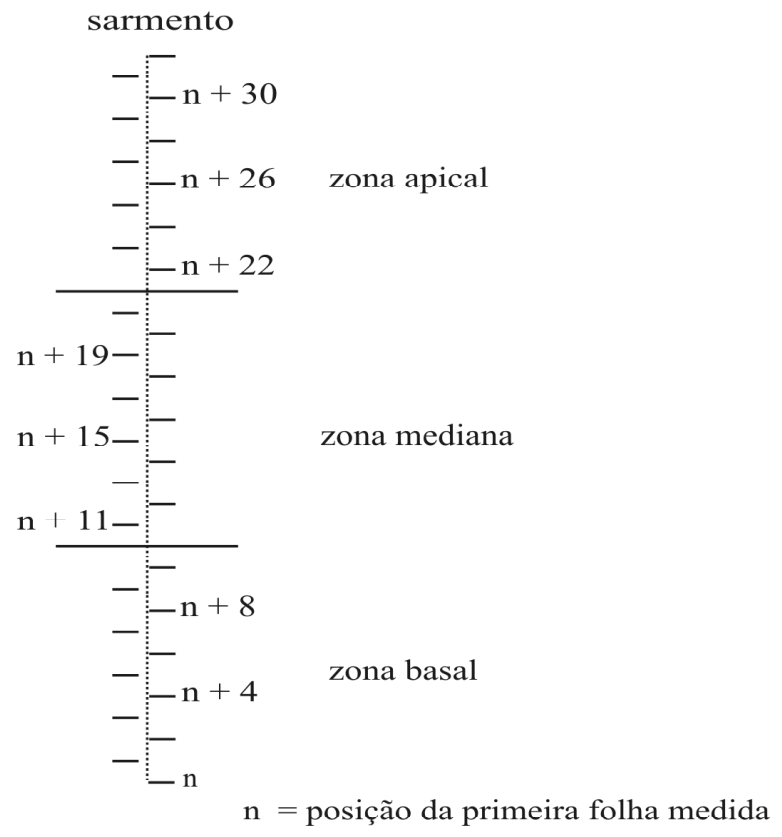

Figura 2 - Esquema representativo para medição da soma das nervuras laterais (L2) por amostragem das folhas de videira. Fonte: Carbonneau (1976), modificado por Orlando (2002). 
A superfície foliar primária pode ser calculada através da fórmula:

$$
\operatorname{SFP}\left(\mathrm{m}^{2}\right)=\mathrm{CNL} 2 \mathrm{X} \text { NFR X NRP, }
$$

em que:

SFP representa a superfície foliar primária $\left(\mathrm{m}^{2}\right)$;

CNL2 representa o valor médio do comprimento das nervuras L2 (m);

NFPR representa o número de folhas por ramo;

NRPP representa o número de ramos por planta.

B)- Superfície foliar secundária, semelhante à realizada nos ramos primários, mas é feita nos ramos secundários ou "netos", que são emitidos após o desponte, considerando-se apenas folhas com nervuras laterais (L2) (Figura 1). Esse dado fornece importantes informações sobre o vigor das plantas. A metodologia é a mesma proposta por Carbonneau (1976).

A superfície foliar secundária pode ser calculada através da fórmula:

\section{$\mathrm{SFS}=\mathrm{CNL} 2 \mathrm{RS} X \mathrm{X}$ NFRS $\mathrm{X}$ NRS,}

em que:

SFS representa a superfície foliar secundária $\left(\mathrm{m}^{2}\right)$;

CNL2RS representa o comprimento das nervuras L2 dos ramos secundários (netos) $(\mathrm{m})$;

NFRS representa o número de folhas por ramo secundário (neto);

NRS representa o número de ramos secundários.

C)- Superfície foliar total: É o somatório da superfície foliar primária e secundária, considerando áreas das plantas com folhas sombreadas ou não, e pode ser obtida através da fórmula:

$$
\mathrm{SFT}=\mathrm{SFP}+\mathrm{SFS}
$$

em que:

SFT representa a superfície foliar total $\left(\mathrm{m}^{2}\right)$;

SFP representa a superfície foliar primária $\left(\mathrm{m}^{2}\right)$;

SFS representa a superfície foliar secundária $\left(\mathrm{m}^{2}\right)$.

D)- Superfície foliar exposta (SFE): Foi medida nas safras 2004 e 2005, durante as fases fenológicas de final de floração (outubro) e pré-colheita (janeiro); os dados apresentados são médios e esse parâmetro serve como um índice sintético que permite estimar a superfície foliar exposta útil e utilizá-la como medida de potencial qualitativo do sistema de condução adotado. A metodologia empregada foi descrita por Carbonneau (1989). A Figura 3 ilustra a base dos parâmetros morfológicos da vegetação de cada sistema de condução, para o cálculo final da superfície foliar exposta, através da fórmula:

$$
\operatorname{SFE}\left(\mathrm{m}^{2} / \mathrm{m}^{2} \text { solo }\right)=\mathrm{S} \mathrm{X} \mathrm{1/E} \mathrm{X}(1-\mathrm{T} / \mathrm{D}),
$$

em que:

SFE representa a superfície foliar exposta $\left(\mathrm{m}^{2} / \mathrm{m}^{2}\right.$ solo); $\mathrm{S}=\delta[\mathrm{Z}(0+0.5)+\mathrm{WS}\rceil-(1-\delta)[(\mathrm{Z}-\mathrm{WS} / 2)]$ :

E representa o espaçamento entre linhas;

T representa a distância entre o dossel de uma planta e outra; D representa o espaçamento entre plantas na linha de plantio; WS representa a largura do dossel superior; $\mathrm{Z}$ representa a altura do dossel;

$\delta$ representa $2 / 3$ céu "claro" e 1/3 "fechado".
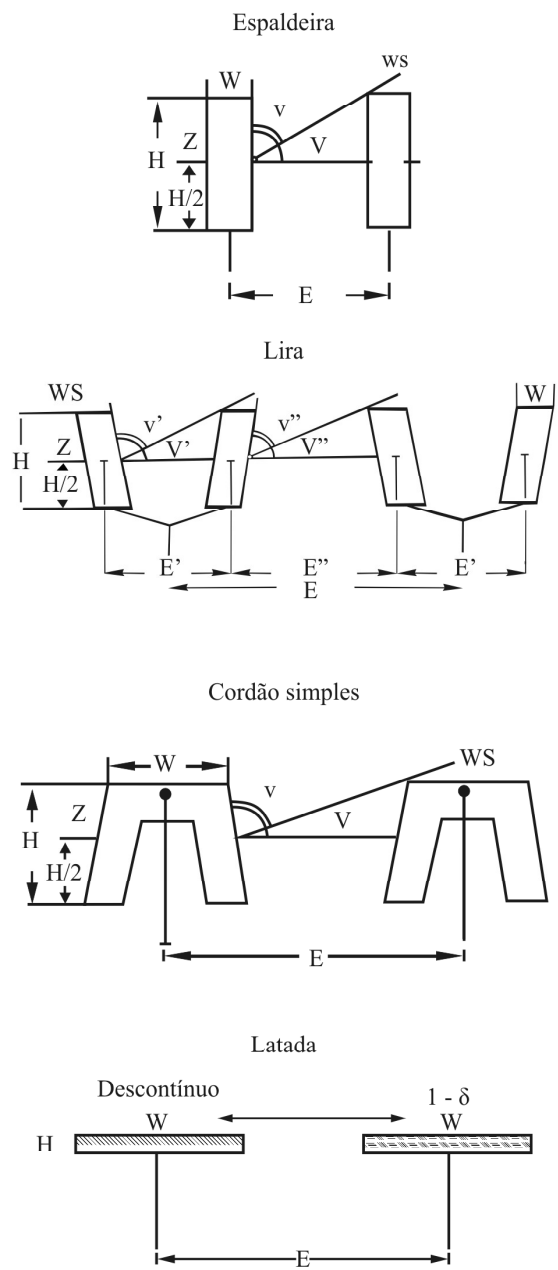

Figura 3 - Caracterização morfológica da vegetação nos sistemas de condução da videira, para cálculo da superfície foliar exposta (SFE), segundo esquema proposto por Carbonneau (1989). 


\section{RESULTADOS E DISCUSSÃO}

\section{Superfície foliar primária, secundária e total}

As superfícies foliares primária (SFP), secundária (SFS) e total (SFT) das variedades 'Folha de Figo' e 'Niágara Rosada', verificadas na média das três safras, são apresentadas na Tabela 1. Os maiores valores de superfície foliar primária foram verificados nos sistemas lira, espaldeira e latada, que se mostraram semelhantes entre si, e o menor valor no cordão simples $\left(4,95 \mathrm{~m}^{2}\right)$. Com relação à superfície foliar secundária, verifica-se que não houve diferenças significativas entre os sistemas lira e espaldeira em ambas variedades. Nota-se que os sistemas cordão simples e latada não apresentaram essas medidas, devido a não realização de desponte nas plantas, como realizado com os sistemas lira e espaldeira, quando as plantas atingiam o ultimo fio de arame. Resultado semelhante ao da superfície foliar primária foi verificado na superfície foliar total, onde também os sistemas lira, espaldeira e latada não apresentaram diferenças significativas entre si, e o sistema cordão simples conferiu o menor valor de superfície foliar primária. Segundo Orlando (2002), o menor comprimento de ramo registrado no sistema cordão simples foi devido ao seu crescimento descendente, o que não ocorre com os demais sistemas de condução. Carbonneau \& Zhang (1989) e Kliewer et al. (2000) reafirmam que a utilização de sistemas de condução que promovam o porte descendente reduz o vigor da planta pela redução da acrotonia.

\section{Superfície foliar exposta (SFE)}

Os dados de superfície foliar exposta das safras 2004 e 2005, bem como os dados médios das duas safras avaliadas nos estágios fenológicos de final de floração e pré-colheita, apresentaram comportamento semelhante para as duas variedades em estudo (Tabela 2). Verificou-se na safra 2004 e 2005, tanto para a variedade 'Folha de Figo' quanto para a 'Niágara Rosada', em ambas as fases fenológicas, que o sistema de condução latada conferiu os maiores valores de SFE, com 1,03 $\mathrm{m}^{2}$ por planta e $1,20 \mathrm{~m}^{2}$ para safra 2004 e $1,14 \mathrm{~m}^{2}$ e $1,26 \mathrm{~m}^{2}$ para safra 2005 , respectivamente. $\mathrm{O}$ sistema lira veio em seguida, com 0,97 $\mathrm{m}^{2}$ e $1,07 \mathrm{~m}^{2}$ na safra 2004 e $1,06 \mathrm{~m}^{2}$ e $1,17 \mathrm{~m}^{2}$ em 2005 para 'Folha de Figo' e 'Niágara Rosada', respectivamente, e por último estão os sistemas espaldeira e cordão simples, com os menores valores de superfície foliar exposta, porém sem diferir estatisticamente um do outro, excetuando-se a 'Niagara Rosada' na safra 2004 (Tabela 2). Uma possível explicação para o resultado verificado é a de que o sistema de condução latada, apesar de conferir sempre os maiores valores de superfície foliar, não teve seu dossel vegetal demasiadamente denso, em razão do baixo vigor do conjunto copa/porta - enxerto empregado, resultando em poucas camadas foliares, o que pode ter contribuído para uma maior superfície foliar exposta.

Carbonneau (1989) relata em seus estudos, que a lira apresenta maior superfície foliar exposta, porque possui dois planos oblíquos de vegetação pouco densa, contribuindo para uma maior exposição aos raios solares. Porém, verificou-se que devido ao crescimento dos ramos nesse sistema, ser ascendente, houve uma maior sobreposição de folhas e por conseqüência, uma menor superfície foliar exposta, razão pela qual em nosso estudo o sistema latada apresentou maior superfície foliar exposta em função da baixa sobreposição da folhagem.

Schneider et al. (1989) acrescentam que a superfície foliar exposta (SFE) é um critério utilizado para medir, indiretamente, o microclima luminoso incidente sobre o dossel vegetativo, juntamente com o índice foliar, não importando a forma da cobertura, permitindo, assim, comparar diferentes sistemas de condução e que permite

Tabela 1 - Superfície foliar primária (SFP), secundária (SFS) e total (SFT) de Videira, em m² por planta, das variedades 'Folha de Figo' e 'Niágara Rosada' conduzidas sob diferentes sistemas de condução.Média das três safras, na fase fenológica de final de maturação "Pintor". EPAMIG, Caldas-MG, 2005.

\begin{tabular}{|c|c|c|c|c|c|c|}
\hline \multicolumn{7}{|c|}{ Dados Médios das safras 2003, 2004 e 2005} \\
\hline \multirow{2}{*}{$\begin{array}{l}\text { Sistema de } \\
\text { Condução }\end{array}$} & \multicolumn{3}{|c|}{ 'Folha de Figo' } & \multicolumn{3}{|c|}{ 'Niágara Rosada' } \\
\hline & $\operatorname{SFP}\left(\mathrm{m}^{2}\right)$ & $\operatorname{SFS}\left(\mathrm{m}^{2}\right)$ & SFT $\left(\mathrm{m}^{2}\right)$ & $\operatorname{SFP}\left(\mathrm{m}^{2}\right)$ & $\operatorname{SFS}\left(\mathrm{m}^{2}\right)$ & $\operatorname{SFT}\left(\mathrm{m}^{2}\right)$ \\
\hline C. simples & $4,95 \quad b$ & & $4,95 \mathrm{~b}$ & $4,06 \quad b$ & & $4,06 \quad b$ \\
\hline Lira & 6,41 & $0,54 \quad \mathrm{a}$ & 6,98 a & $9,07 \quad \mathrm{a}$ & $0,72 \mathrm{a}$ & $9,86 \quad \mathrm{a}$ \\
\hline Espaldeira & 7,28 & $0,54 \quad \mathrm{a}$ & 7,82 a & 10,17 a & $0,53 \mathrm{a}$ & 11,09 a \\
\hline Latada & 7,59 & & 7,59 a & $8,51 \quad \mathrm{a}$ & & 8,51 a \\
\hline
\end{tabular}

*Médias seguidas pela mesma letra na coluna não diferem estatisticamente entre si pelo teste de Scott-Knott, ao nível de 5\% de probabilidade. 
Tabela 2 - Superfície foliar exposta (SFE) de Videira, média das variedades 'Folha de Figo' e 'Niágara Rosada', nas fases fenológicas de final de floração e pré-colheita, conduzidas sob diferentes sistemas de condução na safra 2004, 2005, e valores médios das safras 2004 e 2005, EPAMIG, Caldas-MG, 2005.

\begin{tabular}{lcc}
\hline \multicolumn{1}{c}{ Sistema de Condução } & 'Folha de Figo' & 'Niágara Rosada' \\
\hline & SFE $\left(\mathrm{m}^{2}\right)$ & SFE $\left(\mathrm{m}^{2}\right)$ \\
\hline Espaldeira & Safra 2004 & $0,79 \mathrm{~d}$ \\
Cordão Simples & $0,74 \mathrm{~b}$ & $0,92 \mathrm{c}$ \\
Lira & $0,84 \mathrm{~b}$ & $1,07 \mathrm{~b}$ \\
Latada & $0,97 \mathrm{a}$ & $1,20 \mathrm{a}$ \\
\hline & $1,03 \mathrm{a}$ & \\
\hline Espaldeira & Safra 2005 & $0,84 \mathrm{c}$ \\
Cordão Simples & $0,74 \mathrm{c}$ & $0,85 \mathrm{c}$ \\
Lira & $0,76 \mathrm{c}$ & $1,17 \mathrm{~b}$ \\
Latada & $1,06 \mathrm{~b}$ & $1,26 \mathrm{a}$ \\
\hline & $1,14 \mathrm{a}$ & \\
\hline Espaldeira & $0,74 \mathrm{~b}$ & $0,82 \mathrm{c}$ \\
Cordão Simples & $0,80 \mathrm{~b}$ & $0,89 \mathrm{c}$ \\
Lira & $1,02 \mathrm{a}$ & $1,12 \mathrm{~b}$ \\
Latada & $1,09 \mathrm{a}$ & $1,23 \mathrm{a}$ \\
\hline
\end{tabular}

* Médias seguidas pela mesma letra na coluna não diferem estatisticamente entre si pelo teste de Scott-Knott, ao nível de 5\% de probabilidade.

estimar a superfície foliar exposta útil, sendo possível utilizar esse dado como uma avaliação do potencial qualitativo do sistema de condução.

Com relação aos dados médios das safras 2004 e 2005 (Tabela 2), o sistema latada também conferiu os maiores valores de SFE, com 1,09 $\mathrm{m}^{2}$ na variedade 'Folha de Figo' e 1,23 m² na variedade 'Niágara Rosada', porém sem diferir da lira, com 1,02 $\mathrm{m}^{2}$ na variedade 'Folha de Figo' e diferindo desse sistema de condução, com $1,12 \mathrm{~m}^{2}$, na 'Niagara Rosada'. Todavia, os menores valores de SFE foram verificados nos sistemas espaldeira e cordão simples para as duas variedades, semelhantemente ao verificado nos dados das safras 2004 e 2005 em separado, em que também foram inferiores e não apresentaram diferenças significativas entre si.

\section{CONCLUSÕES}

Os resultados obtidos nesse trabalho permitiram concluir que:

- o sistema cordão simples confere menor superfície foliar total em ambas as variedades;

- a superfície foliar exposta (SFE) é superior nos sistemas de condução latada e lira.

\section{REFERÊNCIAS BIBLIOGRÁFICAS}

CARBONNEAU, A. Etude écophysiologique dês principaux systèmes de conduite interêt qualitatif et économique des vignes en Lyre: premières indications de leur comportement en situation de vigueur élevée. In: CONGRESSO BRASILEIRO DE VITICULTURA E ENOLOGIA, 6., 1990, Bento Gonçalves. Anais... Bento Gonçalves: Embrapa/CNPUV/ABTE/OIV, 1991. p. $21-34$.

CARBONNEAU, A. Apports biologiques récents à l'étude des systèmes de conduite. Bulletin de l'O.I.V., Paris, v. 55, n. 614, p. 273-285, 1982.

CARBONNEAU, A. Interet et codification du systeme de conduite em double palissage-lyre. Compte Rendu, Bordeaux, n. 4, p. 202-214, 1989.

CARBONNEAU, A. Analyse de la croissance des feuilles du sarment de vigne: estimation de la surface foliare par enchantillonnage. Connaissance Vigne Vin, Bordeaux, v. 10, n. 2, p. 141-159, July 1976. 
CARBONNEAU, A.; ZHANG, D. P. Influence of winter pruning methods on grapevine physiology: consequences for mechanical pruning and justifications of the principle of the: "alternated crenel": originality of sap flow measurements in the trunk. Rivista di Ingegnere, Milano, v. 9, p. 121-132, 1989.

CASTRO, R. Sistemas de condução da vinha: evolução, tendências atuais e estudos a decorrer em Portugal. In: SIMPÓSIO DE CIÊNCIA E TECNOLOGIA EM VITICULTURA, 1., 1989, Dois Portos. Anais... Dois Portos : [s.n.], 1989.

CHAMPAGNOL, F. Éléments de physiologie de la vigne et de viticulture générale. Montpellier: Déhan, 1984. 351 p.

KLIEWER, W. M.; WOLPET, J. A.; BENZ, M. Trellis and vine spacing effects on growth, canopy microclimate, yield and fruit composition of Cabernet Sauvignon. Acta Horticulturae, Wageningen, v. 526, p. 21-31, 2000.
NOGUEIRA, D. J. P. Porta-enxertos de videira. Informe Agropecuário, Belo Horizonte, v. 10, n. 117, p. 22-24, set. 1984.

ORLANDO, T. G. S. Características ecofisiológicas de cultivares de videira em diferentes sistemas de condução. 2002. 126 p. Tese (Doutorado em Fitotecnia) - Universidade Federal de Lavras, Lavras, 2002.

POMMER, C. V.; PASSOS, I. R. S.; TERRA, M. M.; PIRES, J. P. Variedades de videira para o Estado de São Paulo. Campinas: IAC, 1997. 59 p. (Boletim técnico, 166).

SCHNEIDER, C.; ANCEL, J.; HEYWANG, M. Etude comparative entre plusiers systèmes de conduite sur deux cepages d'Alsace. Compte Rendu, Bordeaux, n. 4, p. 97-108, 1989.

SMART, R. E. Principles of grapevine canopy microclimate manipulation with implications for yield and quality: a review. American Journal of Enology and Viticulture, Davis, v. 36, n. 3, p. 230-239, 1985. 